Artículos

\title{
Formación de cultura ambiental desde el enfoque de ciclo de vida: una propuesta
} pedagógica para la sostenibilidad*

Formation of Environmental Culture under the Life Cycle Approach: A Pedagogical Proposal for Sustainability

Paola Andrea Calderón Cuartas ${ }^{\text {a }}$

Universidad Católica de Manizales, Colombia

pcalderon@ucm.edu.co

ORCID: http://orcid.org/0000-0003-0611-8299

Wilmar Osorio Viana

Universidad Católica de Manizales, Colombia

ORCID: http://orcid.org/0000-0002-1223-3472

Fecha de recepción: 01 Septiembre 2018

Fecha de aprobación: 27 Octubre 2018

Fecha de publicación: 30 Junio 2019

Javier Mauricio Naranjo Vasco

Universidad Católica de Manizales, Colombia

ORCID: http://orcid.org/0000-0001-9806-4878

Tomás De Jesús Guzmán Hernández

Instituto Tecnológico de Costa Rica, Costa Rica

ORCID: http://orcid.org/0000-0002-2719-8550

\section{Resumen:}

Con el objetivo de analizar el potencial de la articulación entre el enfoque de ciclo de vida y la educación ambiental para generar procesos formativos que arraiguen a mediano plazo una cultura ambiental, se realizó un ejercicio reflexivo de carácter exploratoriodescriptivo que relacionó tres temáticas: la producción y el consumo sostenible, el enfoque de ciclo de vida y la educación ambiental. De esta forma se llevó a cabo un desarrollo teórico sobre la importancia del enfoque de ciclo de vida para la toma de consciencia ambiental, que permita el trabajo individual y colectivo de diferentes actores sociales e institucionales en la prevención y resolución de los problemas ambientales que surgen por los procesos insostenibles de producción, consumo y posconsumo. Se concluye que la articulación entre el enfoque de ciclo de vida y la educación ambiental es clave para la configuración de estrategias pedagógicas que den origen a procesos participativos de gestión ambiental basados en una cultura ambiental a nivel territorial.

Palabras clave: cultura ambiental, desarrollo sostenible, deterioro ambiental, educación ambiental, enfoque de ciclo de vida, producción y consumo sostenible, sociedad-naturaleza.

\section{Abstract:}

In order to analyze the potential of articulation between the life cycle approach and the environmental education to produce formation processes to create an ingrained environmental culture in the medium term, an exploratory-descriptive reflective exercise was carried out relating three themes: Sustainable production and consumption, life cycle approach, and environmental education. This way, a theoretical development was about the significance of the life cycle approach to raise environmental awareness, which would allow both individual and collective work by different social and institutional actors for the prevention and solution of environmental problems arising from unsustainable processes of production, consumption and post-consumption. The conclusion is drawn that the articulation between the life cycle approach and the environmental education is key for shaping pedagogical strategies to produce, in turn, participatory environmental management processes based on an environmental culture working in the territory.

Keywords: environmental culture, sustainable development, environmental deterioration, environmental education life cycle approach, sustainable production and consumption, society-nature.

Notas de autor

\footnotetext{
a Autora de correspondencia. Correo electrónico: pcalderon@ucm.edu.co
} 


\section{Introducción}

Los retos impuestos por los Objetivos de Desarrollo Sostenible (ODS) y el Acuerdo sobre Cambio Climático para frenar el deterioro ambiental a nivel territorial implican abordar las problemáticas ambientales desde un enfoque integral y preventivo. Las metas fijadas por la Agenda 2030 en sus ODS 4, 11, 12 y 13 plantean de manera implícita la necesidad de articular el enfoque de ciclo de vida (ECV) y la educación ambiental (EA), de modo que permita la consolidación de una cultura ambiental que sustente la transformación de ciudades y comunidades hacia la sostenibilidad.

En Colombia, la Política de Gestión Ambiental Urbana (Ministerio de Ambiente y Desarrollo Sostenible [MAVDT], 2008), la Política Nacional de Producción y Consumo Sostenible (MAVDT, 2010) y la Política Nacional para la Gestión Integral de Residuos (Consejo Nacional de Política Económica y Social [CONPES] y Departamento Nacional de Planeación [DNP], 2016) han fundamentado lineamientos y estrategias desde el ECV y la economía circular.

El ECV permite identificar todas las fases del proceso de generación de un bien o servicio (desde la extracción de las materias primas hasta el fin de su vida útil).

[Los ECV] son útiles ya que permiten identificar tanto las oportunidades como los riesgos de un producto o tecnología nueva, desde la materia prima hasta el proceso de desecho. Existe una gama de enfoques de ciclo de vida que va desde lo cualitativo (el concepto del ciclo de vida) hasta lo exhaustivamente cuantitativo (el análisis del ciclo de vida). (Programa de las Naciones Unidas para el Medio Ambiente [PNUMA], 2004, p. 7)

El ECV lleva implícito un propósito esencial que se centra en la toma de consciencia, en la responsabilidad y el compromiso para trabajar individual y colectivamente en la prevención y resolución de los problemas ambientales que surgen de los procesos de transformación de materia prima en mercancías y su posterior desecho. En este sentido, el ECV implica un proceso de EA.

De la articulación entre el ECV y la EA emergen las estrategias pedagógicas que posibiliten la comprensión, explicación e integración de los procesos de producción, consumo y posconsumo. Además, genera una perspectiva integrada de los impactos sociales, económicos y ambientales de estos tres procesos, los cuales a su vez dependen de las decisiones asumidas por los ciudadanos en sus diferentes roles: consumidores, empresarios, comerciantes, gobiernos.

Esta articulación permite a los actores sociales e institucionales una toma de decisiones consiente y consistente en situaciones cotidianas, tales como hacer las compras, crear el diseño de un producto nuevo o formular una política gubernamental con criterios de sostenibilidad, de manera que se fortalezca la responsabilidad individual y colectiva con lo ambiental (PNUMA, 2004).

Este artículo tiene como objetivo analizar el potencial de la articulación entre el ECV y la EA, para generar procesos formativos, formales y no formales, que arraiguen a mediano plazo una cultura ambiental, que favorezca una relación equilibrada entre sociedad y naturaleza desde un pensamiento crítico y una ética que fundamente valores y comportamientos para con lo ambiental y sus modos de apropiación:

La educación para el desarrollo sostenible ${ }^{[1]}$ demanda nuevas orientaciones y contenidos; nuevas prácticas pedagógicas donde se plasmen las relaciones de producción de conocimientos y los procesos de circulación, transmisión y diseminación del saber ambiental. Esto plantea la necesidad de incorporar valores ambientales y nuevos paradigmas del conocimiento en la formación de nuevos actores de la educación ambiental y el desarrollo sostenible. ${ }^{[2]}$ (Leff, 1998, p. 213)

Una relación equilibrada de la sociedad con la naturaleza es la que se conoce como cultura ambiental, la cual valora y reconoce plenamente la esfera no humana y nuestra dependencia de ella, de modo que oriente una adecuada toma de decisiones, que minimice los impactos sobre la biosfera (Plumwood, 2005). Una relación equilibrada se basa en el principio de economía circular o cierre del ciclo para la conservación y uso sostenible de los recursos, la minimización de la generación de residuos, la recuperación de materiales y la eficiencia 
energética, manteniendo el mayor tiempo posible los materiales y la energía en el ciclo productivo (Jouni, Honkasalo y Seppälä, 2018).

Esta relación propone superar el esquema tradicional de extraer-producir-usar-desechar, el cual predomina actualmente en los países de América Latina y el Caribe, reconocido como economía lineal (PNUMA, 2018). Un cambio de paradigma, que más allá de herramientas técnicas y de infraestructura, requiere una toma de consciencia, así como las actitudes y las aptitudes para transformar la actual situación de deterioro ambiental.

\section{Metodología}

Este artículo de reflexión es de carácter exploratorio-descriptivo; exploratorio porque se basó en una revisión del estado del arte de tres categorías conceptuales claves, abordadas y analizadas desde diferentes autores: la producción y el consumo sostenible, el ECV y la EA; y descriptivo, porque caracterizó y relacionó las categorías conceptuales desde una perspectiva reflexiva, analizando la importancia del ECV para generar estrategias pedagógicas de EA que se orienten hacia la toma de consciencia sobre el conjunto de los impactos ambientales que conllevan los procesos productivos y de consumo, y con ello contribuir a la consolidación de una cultura ambiental, pilar del desarrollo sostenible.

La reflexión se desarrolla esencialmente a partir de la revisión y análisis de fuentes secundarias de información: libros, documentos, artículos y otros recursos, desde los cuales se estructuró una reflexión profunda, que se sustenta además en la amplia experiencia de los autores como formadores en instituciones educativas.

El ejercicio reflexivo se contextualiza en la actual situación de deterioro ambiental, en la que se hace evidente la necesidad de generar procesos efectivos de gestión y educación ambiental, en el marco de las tendencias mundiales en ECV, reconociendo su potencial para ayudar a la toma de decisiones ambientalmente responsables, propósito final de la EA.

\section{Resultados}

\section{El deterioro ambiental y la necesidad de un desarrollo sostenible}

El desequilibrio en la relación entre sociedad y naturaleza se manifiesta principalmente en el deterioro de la calidad ambiental de los territorios urbanos y de la salud pública, lo que llama la atención de los colectivos sociales hacia esta problemática de carácter global. Este desequilibrio ha sido creado por la idea generalizada del crecimiento económico basado en la transformación de recursos naturales en bienes y servicios, la cual ha establecido una dinámica económica que desborda y supera cada vez más los límites planetarios.

El concepto de límites planetarios es un marco integral útil para ilustrar los peligros de la interferencia humana en el sistema de la Tierra, interferencia que ejerce el ser humano a través de modalidades insostenibles de consumo y producción. Respetar los límites de la Tierra implica conocer cómo las sociedades humanas pueden desarrollarse y progresar de manera segura, lo que requiere la comprensión humana del funcionamiento y la resiliencia de los ecosistemas globales. (World Wildlife Fund [WWF], 2018)

Y es tan evidente el deterioro ambiental global que cada vez es más aceptada la teoría del Antropoceno, una nueva era geológica generada por la huella ecológica de la especie Homo sapiens. Cambios acelerados en los últimos cincuenta años, como el aumento significativo en la temperatura media global, la acidificación de los océanos, la pérdida masiva de biodiversidad y las emisiones de carbono, son algunos de los más graves impactos ambientales asociados a la actividad antropogénica (Waters et al., 2016). 
Este deterioro se agudiza cuando los intereses económicos ven a la naturaleza y a lo ambiental exclusivamente como materia prima, la cual transformada en bienes y servicios fortalece el sistema financiero y empobrece el sistema ambiental, que se reduce a ser fuente de recursos y vertedero de residuos:

La degradación ambiental emerge del crecimiento y la globalización de la economía. Esta escasez generalizada no solo se manifiesta en la degradación de las bases de sostenibilidad ${ }^{[3]}$ ecológica del proceso económico, sino como una crisis de civilización que cuestiona la racionalidad del sistema social, los valores, los modos de producción y los conocimientos que lo sustentan. (Leff, 1998, p. 4)

Por ello, esta problemática ha suscitado desde hace más de medio siglo el debate político de líderes mundiales acerca del surgimiento de iniciativas, declaraciones y lineamientos que pretenden conjugar la utilidad de los recursos naturales con el crecimiento económico.

Propuestas teóricas y políticas, como el ecodesarrollo y el desarrollo sostenible, han planteado la urgencia de instaurar nuevos modos de producción y de consumo desde alternativas sostenibles. El ecodesarrollo fue una propuesta pionera, realizada por Maurice Strong en 1973, con la intención de conseguir un desarrollo que logre un crecimiento económico que considere los límites de la naturaleza y las potencialidades ecológicas de cada región:

El desarrollo, entendido como Ecodesarrollo, o sea un intento de establecer relaciones mutuamente beneficiosas y sostenibles entre una población humana y el ecosistema de que forma parte, presupone regulación del tamaño, del ritmo de crecimiento, y de la distribución de las poblaciones según las capacidades del ecosistema, que varían, lógicamente, con el nivel tecnológico existente. (Clinton, 1975 , p. 18)

Sin embargo, el ecodesarrollo no logró vencer las barreras del manejo sectorial, y sus estrategias no consiguieron superar la resistencia del orden económico, lo que generó la disolución de la capacidad crítica y la visión transformadora de las prácticas ambientales que lo estructuraron. El crecimiento económico siempre ha sido una prioridad para las políticas gubernamentales, de manera que se han configurado programas neoliberales agresivos con respecto a la apropiación de la naturaleza, los cuales han complejizado paulatinamente la problemática ambiental: "En ese momento empieza a caer en desuso el discurso del Ecodesarrollo y a ser suplantado por el discurso de Desarrollo Sostenible” (Leff, 1998, p. 18); esta es precisamente la génesis del desarrollo sostenible, que proyecta un equilibrio "capaz de ecologizar la economía, eliminando la contradicción entre el crecimiento económico y la conservación de la naturaleza” (Leff, 1998, p. 17).

Por su parte, el desarrollo sostenible ha sido presentado desde 1987 por el informe Nuestro Futuro Común o Informe Brundtland, ratificado posteriormente por múltiples protocolos internacionales, como la Agenda 21 (1992), la Carta de la Tierra (2000), los Objetivos de Desarrollo del Milenio (2000), el informe El Futuro que Queremos (2012) y los Objetivos de Desarrollo Sostenible (2015). Estos protocolos han generado directrices acerca de la necesidad de avanzar hacia la satisfacción de las necesidades humanas desde un crecimiento económico equitativo, garantizando la conservación ecosistémica para las generaciones futuras.

El concepto de ambiente adquiere así un significado estratégico, tanto en lo político como en lo económico: en lo político, porque la problemática ambiental se convierte en una temática fundamental de las agendas de los gobiernos, y en lo económico, porque, sin negar el carácter productivo, prefiere un crecimiento económico equitativo, que supere la concepción de lo ambiental desde la explotación económica de la naturaleza y la distribución inequitativa de los costos ecológicos. Desde allí toma importancia la EA:

Si la sostenibilidad del planeta requiere la desmaterialización del consumo, es en esos escenarios adonde se debería dirigir la educación ambiental informal. El ambientalismo debería tomar consciencia y retornar a sus orígenes territoriales, ecológicos, aquellos que le demuestran instantáneamente y en cualquier lugar la realidad y la validez de su misión. (Carrizosa, 2014, p. 232) 


\section{Los sistemas de producción y consumo para las ciudades sostenibles}

El verdadero reto del desarrollo sostenible es asegurar un crecimiento económico sostenido que garantice la satisfacción de las necesidades humanas presentes y futuras, sin detrimento de la calidad ambiental.

A partir de la declaración Transforming our world: The 2030 agenda for sustainable development (United Nations [UN], 2015), se proponen alternativas razonables desde un enfoque integrado y práctico del desarrollo, entendido como la producción y el consumo sostenibles (PyCS):

La producción y uso de bienes y servicios que responde a las necesidades básicas y aporta una mejora calidad de vida, mientras minimiza el uso de recursos naturales, materiales tóxicos y emisiones de desechos y contaminantes sobre todo el ciclo de vida de los bienes y servicios. Así, no pone en riegos la capacidad de satisfacer las necesidades de las generaciones futuras. (Ministerio de Medio Ambiente de Noruega, 1994, citado en PNUMA, 2008, p. 19)

La integración de los sistemas de producción y consumo en el marco de las ciudades y comunidades sostenibles plantea retos y esfuerzos importantes para todos los países del mundo, especialmente para los miembros de América Latina y el Caribe (ALC), referidos en la V Reunión del Consejo de Expertos de Gobierno en PyCS ${ }^{[4]}$ para ALC:

a. Políticas y estrategias nacionales en PyCS: implica fortalecer los procesos de información, educación y capacitación a la población en esta materia y dar prioridad y fomentar una mayor responsabilidad social y ambiental de las empresas entre los sectores productores de bienes y consumo masivo que generan mayores impactos ambientales y sociales [...].

b. Las pequeñas y medianas empresas: implica priorizar sectores a nivel subregional vinculados a los servicios ecosistémicos y crear o fortalecer mecanismos e instrumentos económicos que apoyen la sostenibilidad de los sectores productivos [...].

c. Las compras públicas sostenibles: implica adoptar una estrategia de aplicación gradual para la incorporación de criterios ambientales y sociales en los procesos de contratación de bienes y servicios prioritarios [...].

d. Estilos de vida sostenibles: implica ampliar la participación e involucrar en la acción a toda la comunidad en el desarrollo y ejecución de acciones para promover estilos de vida sostenibles. (PNUMA, 2010, pp. 34, 35)

Colombia ha realizado algunos avances en estos temas, específicamente desde la formulación de políticas públicas que deben implementarse de manera articulada, las cuales han introducido el ECV al país y plantean un cambio de comportamiento de los diferentes actores que permita avanzar hacia una economía circular: "La capacidad básica para construir una primera concientización sobre PyCS que propicie el cambio de comportamientos se genera mediante la EA en las instituciones educativas y las asociaciones de consumidores" (MAVDT, 2010, p. 32).

En la figura 1, se presentan las principales estrategias propuestas en algunas de las políticas colombianas para la formación de cultura ambiental. 


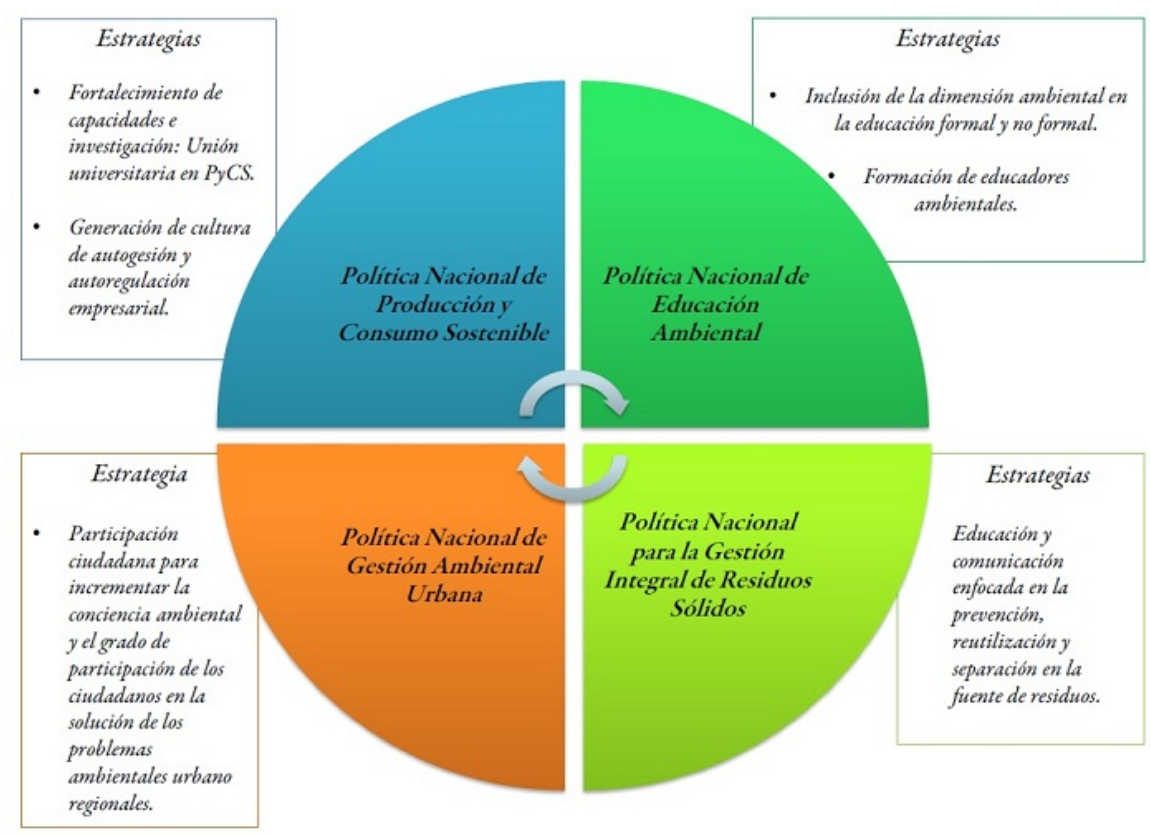

FIGURA 1.

Políticas colombianas y sus estrategias para la formación de cultura ambiental Fuente: elaboración propia, con base en CONPES y DNP (2016); MAVDT (2008,

2010); Ministerio del Medio Ambiente y Ministerio de Educación Nacional (2002)

Es así como la perspectiva integral con la que fue concebida la política de PyCS permite trascender los modos de apropiación tradicional de la naturaleza, desde una comprensión sistémica y compleja de la realidad:

Se trata de repensar las formas tradicionales sobre productos, sus funciones y mercados y los impactos ambientales que ocurren a lo largo de sus ciclos de vida [...]. Otra estrategia de producción y consumo inteligente se dirige al cierre de los ciclos de materiales hacia cero emisiones, mediante la prevención en la generación de desperdicios, la reducción al mínimo en el uso de materiales e insumos y el aprovechamiento de los residuos. (MAVDT, 2010, p. 29)

De esta manera, la PyCS ha surgido en Colombia como un binomio, que ha traído consigo alternativas sostenibles aún incipientes, como la producción más limpia, la ecoinnovación, las energías renovables no convencionales, el consumo sostenible, las compras responsables, la ecologística y la gestión posconsumo. Estas alternativas, concebidas de manera articulada, dan vida y hacen factible en el tiempo la aplicación de un ECV, que considere todas las fases del ciclo productivo, relacionando integradamente sus aspectos ambientales y sus posibles impactos.

\section{Enfoque de ciclo de vida en la educación y la cultura ambiental}

\section{Lo interesante del ECV en el marco de las ciudades sostenibles es}

que aborda la totalidad de las actividades relacionadas con un producto o servicio hasta su disposición final, incluyendo la investigación y el desarrollo, la extracción y el procesamiento de materias primas, la fabricación, el transporte y la distribución, la utilización, reutilización y mantenimiento, y el reciclaje y la eliminación final. (Rovira, Patiño y Schaper, 2017, p. 20)

Las finalidades del ECV establecen una relación directa con los procesos de formación de cultura ambiental, pues, además de ilustrar sobre las relaciones entre los procesos de producción, consumo y posconsumo, este enfoque transmite y difunde principios y valores de cada contexto ambiental.

A su vez, la EA se convierte en una herramienta para la sostenibilidad: 
El primero de los elementos que determinan que una educación sea ambiental es que esté expresamente enmarcada en un sistema de valores y que reconozca como su objetivo fundamental promover la apropiación y aplicación de esos valores en todos los actos de la vida cotidiana. Entre esos valores, que a la vez constituyen los requisitos y el propósito último de la sostenibilidad, se destacan: [...] La comprensión de la realidad como un sistema complejo, producto de múltiples interacciones, y la capacidad para entender y asumir la sostenibilidad como un proceso, dependiente tanto de las decisiones humanas como de nuestra capacidad para sincronizarnos con los ciclos de la naturaleza. (Wilches-Chaux, 2008, p. 46)

La sostenibilidad requiere de una EA que contribuya a la formación de una cultura ambiental que oriente la acción humana desde una ética ambiental y un pensamiento crítico y propositivo que permita reconfigurar el equilibrio de la relación entre sociedad y naturaleza (figura 2). Las estrategias pedagógicas asumidas desde un ECV deberán fortalecer la participación activa y vinculante de los diferentes actores para la toma de decisiones desde las condiciones propias de las comunidades y medios de vida de las poblaciones.

Estas estrategias tienen como finalidad, redefinir y resignificar los procesos de formación ambiental contextualizados en las problemáticas que surgen desde los ciclos de vida, de manera que se superen las formas tradicionales de abordar la problemática ambiental, por medio de una pedagogía que se constituya desde una concepción holística e interdisciplinaria, integradora de saberes y conocimientos, técnicas y prácticas de producción y consumo sostenibles.

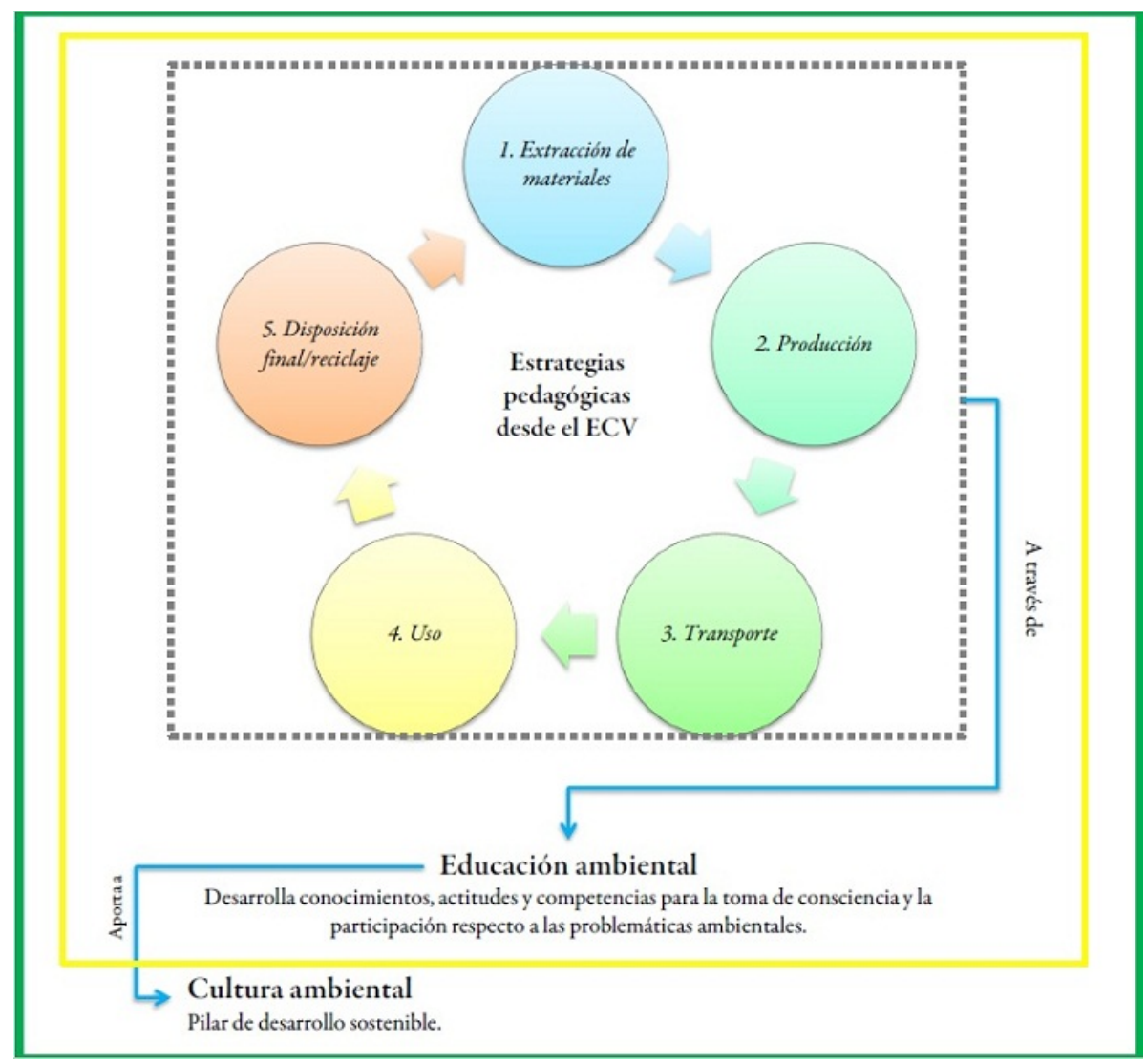

FIGURA 2.

Enfoque de ciclo de vida y su relación con la educación y la cultura ambiental Fuente: elaboración propia

\section{Pedagogía ambiental para la aplicación de un enfoque de ciclo de vida}

La finalidad de las estrategias pedagógicas que surgen de la articulación entre el ECV y la EA fundamentan un saber que concibe el ambiente desde principios y valores que determinan prácticas sostenibles; un saber que 
impulse la transformación de modalidades de producción y consumo para la consolidación de una cultura ambiental.

Estas estrategias permiten ampliar las alternativas de solución de los impactos ambientales negativos generados por la extracción de las materias primas y su transformación, distribución y uso.

"Los valores inadecuados en la relación Sociedad-Naturaleza, las prácticas insostenibles de uso de recursos naturales, la incipiente educación ambiental, los patrones intensivos de consumo, la poca recuperación y difusión de prácticas de producción ambientalmente sostenibles y la ideología dominante recursointensiva" (Perez et al., 2008, p. 64) son los determinantes culturales de la problemática ambiental en Colombia que se presentan como imperativos, retos a los cuales deben responder las estrategias pedagógicas.

Las estrategias pedagógicas que surgen desde el ECV y que proyectan una PyCS refieren una praxis colectiva desde principios de responsabilidad social y ética ambiental. Esta praxis deberá evidenciarse en el territorio, en procesos extractivo-mineros que sean social y ambientalmente más responsables, en procesos de urbanización que respeten la estructura ecológica de las ciudades, en la oferta de productos sostenibles (incluyendo su forma de distribución), en compras individuales e institucionales con criterios de sostenibilidad, en las cuales prime el apoyo a las economías locales, nacionales y regionales y el comercio justo.

Estas estrategias implicarán además la reducción de residuos sólidos, vertimientos y emisiones y el aumento del reciclaje, de modo que se favorezca la consolidación de comunidades con estilos de vida sostenibles que privilegien la protección de la vida y la prevención de la contaminación ambiental.

Una EA para la formación de cultura ambiental desde el ECV considera importante concebir y desarrollar procesos pedagógicos para suscitar la transformación de comportamientos individuales y colectivos, a partir del conocimiento de las relaciones entre los modos de apropiación y consumo que tiene un producto o servicio en cada fase de su ciclo de producción y uso:

El aprendizaje para el consumo sostenible apunta en primer lugar a desarrollar conocimientos y competencias para la toma de decisiones informadas sobre el consumo. No es solo el conocimiento fáctico ("el saber que") sino también el conocimiento procedimental ("el saber cómo") lo que está en juego. (Adomßent, Barth, Fischer, Richter y Rieckmann, 2014, p. 4)

En este sentido, es crucial comprender y transformar el comportamiento humano para avanzar hacia procesos de desarrollo sostenible, más aún cuando existe una brecha comprobada entre las intenciones (lo que las personas piensan hacer) y las acciones (lo que realmente hacen) (Páramo, 2017; United Nations Environment Programme, 2017).

De esta manera, las estrategias pedagógicas se fundamentan en la posibilidad de transformar las concepciones de los sujetos acerca de su realidad y contexto, al permitirles comprender cómo los recursos y productos que consumen tienen una historicidad: un proceso de causas y efectos que condujo finalmente a que estuviera disponible para adquirirlo, usarlo y consumirlo. Esto les permite:

1. Fortalecer sus habilidades analíticas y reflexivas para la reconstrucción propia de sus saberes acerca a las relaciones de causa y efecto de los procesos de producción, consumo y posconsumo.

2. Aplicar su responsabilidad y ética en acciones orientadas según sus propios valores y conocimientos de los modos de apropiación de la naturaleza que dieron origen al recurso/producto.

Finalmente, las estrategias deben articular componentes de formación, investigación y práctica social sobre los sistemas de producción y consumo, y su relación con el desarrollo local, para la educación y la cultura ambiental. Asimismo, estas estrategias deben facilitar el pensamiento crítico y creativo desde una praxis ambiental comprometida, tanto de los estudiantes como de los maestros y de las familias, una praxis que permita que los saberes, conocimientos y competencias trasciendan del entorno escolar hacia la experiencia cotidiana, de suerte que nos permita producir y consumir de una manera responsable y consciente, es decir, sostenible. 


\section{Conclusiones}

La apuesta por la EA requiere de la estructuración de estrategias pedagógicas que permitan la transformación de los procesos de producción, consumo y posconsumo inconscientes y desinteresados con lo ambiental. Pero transformar exige de una ardua labor pedagógico-ambiental que, de una manera $u$ otra, debe estar en contra del derroche y el desperdicio.

Las estrategias pedagógicas que surgen desde el ECV favorecen la comprensión contextualizada de los sistemas de PyCS y posibilitan procesos los participativos y corresponsables para una adecuada gestión ambiental.

Para que estos procesos pedagógico-ambientales sean efectivos, se deberá reconocer la realidad territorial específica e identificar los conocimientos, las actitudes y las competencias que se tienen y se requieren para lograr los objetivos en el corto, el mediano y el largo plazo. Por ello, es necesario formular planes y programas de EA que tengan como propósito lograr cambios en los comportamientos de los diferentes actores sociales que intervienen en el ciclo de vida, los cuales deben asumir decisiones ambientalmente responsables.

El contexto político-normativo actual de Colombia es propicio para desarrollar procesos pedagógicoambientales basados en el ECV que permitan la aplicación de alternativas sostenibles propuestas desde un enfoque integrador del desarrollo. En todo caso, se deberán identificar las barreras que limitan la aplicación de estas alternativas, las cuales tienen que ver con características propias del comportamiento humano, socioculturales y del entorno.

La responsabilidad social y ambiental de la educación exhorta a los profesores, los estudiantes y los miembros de las comunidades educativas a que sean persistentes y coherentes en su compromiso con lo ambiental, a partir de una educación coherente que les brinde la posibilidad de actuar de tal manera que lo que piensan y quieren se refleje en sus comportamientos y acciones, de modo que sean éticos y respetuosos con las generaciones presentes y futuras.

\section{Referencias}

Adomßent, M., Barth, M., Fischer, D., Richter, S., y Rieckmann, M. (2014). Learning to change universities from within: A service-learning perspective on promoting sustainable consumption in higher education. Journal of Cleaner Production, 62(1), 72-81. doi: 10.1016/j.jclepro.2013.04.006

Carrizosa, J. (2014). Colombia compleja. Bogotá: Jardín Botánico José Celestino Mutis. Instituto de Investigación de Recursos Biológicos Alexander Von Humboldt. Recuperado de https://www.repository.humboldt.org.co/hand le/20.500.11761/32548

Clinton, R. (1975). Hacia una teoría del ecodesarrollo: concepto clave para ubicar el rol de políticas de población dentro del proceso de desarrollo. Documento presentado en el Seminario sobre Estructura Política y Políticas de Población, Santiago de Chile, 26-30 de mayo. Recuperado de https://www.repositorio.cepal.org/bitstream/han dle/11362/20298/D-00155.01_es.pdf?sequence=1\&isAllowed=y

Consejo Nacional de Política Económica y Social (CONPES), y Departamento Nacional de Planeación (DNP). (2016) Política Nacional para la Gestión Integral de Residuos Sólidos. Documento CONPES 3874. Recuperado de https://www.colaboracion.dnp.gov.co/CDT/Conpes/Económicos/3874.pdf

Jouni, K., Honkasalo, A., y Seppälä, J. (2018). Circular economy: The concept and its limitations. Ecological Economics, 143, 37-46. doi: 10.1016/j.ecolecon.2017.06.041

Leff, E. (1998). Saber ambiental. Sustentabilidad, racionalidad, complejidad, poder. México: Siglo XXI.

Ministerio de Ambiente y Desarrollo Sostenible (MAVDT). (2008). Política de Gestión Ambiental Urbana. Recuperado de https://www.minambiente.gov.co/images/AsuntosambientalesySectorialyUrbana/pdf/Polit\% C3\%ACcas_de_la_Direcci\%C3\%B3n/Politica_de_Gestion_Ambiental_Urbana.pdf 
Ministerio de Ambiente y Desarrollo Sostenible (MAVDT). (2010). Política Nacional de Producción y Consumo. Hacia una cultura de consumo sostenible y transformación productiva. Recuperado de https://www.uniagraria. edu.co/images/union_universitaria/Politica_Nacional_Produccion_Consumo_Sostenible.pdf

Ministerio del Medio Ambiente, y Ministerio de Educación Nacional. (2002). Política Nacional de Educación Ambiental SINA. Recuperado de https://www.cmap.upb.edu.co/rid=1195259861703_152904399_919/poli ti-ca_educacion_amb.pdf

Páramo, P. (2017). Reglas proambientales: una alternativa para disminuir la brecha entre el decir-hacer en la educación ambiental. Suma Psicológica, 24(1), 42-58. doi:10.1016/j.sumpsi.2016.11.001

Perez, M. A., Rojas, J. H., Arias-Arévalo, P., Zabala, M. A., Guhl-Nannetti, E., Peña-Varón, M., ... López Bernal, O. (2008). Hacia el desarrollo sostenible en Colombia. En Senado de la República, Piensa Colombia, documentos de política pública: los aportes de la academia (t. 1, vol. 3, pp. 1-63). Manizales: Universidad Nacional de Colombia.

Plumwood, V. (2005). Environmental culture. The ecological crisis of reason. Londres y Nueva York: Routledge. Recuperado de https://www.epdf.pub/environmental-culture-the-ecological-crisis-of-reason.html

Programa de las Naciones Unidas para el Medio Ambiente (PNUMA). (2004). ¿Por qué adoptar un enfoque de ciclo de vida? Recuperado de https://www.sustainabledevelopment.un.org/content/documents/1731Why_take_a_ life_cycle_approach_ES.pdf

Programa de las Naciones Unidas para el Medio Ambiente (PNUMA). (2008). Planificación para el cambio. Guías para programas nacionales sobre consumo y producción sustentables. Recuperado de https://www.unep.fr/shar ed/publications/pdf/WEBx0157xPA-Planning\%20for\%20ChangeSPA.pdf

Programa de las Naciones Unidas para el Medio Ambiente (PNUMA). (2010). Aquí y ahora. Educación para el consumo sostenible. Recomendaciones y orientaciones. Recuperado de https://www.unep.fr/shared/publicatio ns/pdf/DTIx1255xPA-Here\%20and\%20Now\%20SPA.pdf

Programa de las Naciones Unidas para el Medio Ambiente (PNUMA). (2018). Perspectiva de la gestión de residuos en América Latina y el Caribe. Programa de las Naciones Unidas para el Medio Ambiente Recuperado de: http s://www.wedocs.unep.org/handle/20.500.11822/26448

Rovira, S., Patiño, J., y Schaper, M. (comps.). (2017). Ecoinnovación y producción verde. Una revisión sobre las políticas de América Latina y el Caribe. Cepal-CIID del Canadá. Recuperado de https://www.repositorio.cepal.org/bit stream/handle/11362/40968/1/S1700072_es.pdf

United Nations (UN). (2015). Transforming our world: The 2030 agenda for sustainable development. General Assembly Resolution 70/1. Recuperado de https://www.sustainabledevelopment.un.org/content/documents/ 21252030\%20Agenda\%20for\%20Sustainable\%20Development\%20web.pdf

United Nations Environment Programme. (2017). Consuming differently, consuming sustainably: Behavioural insigths for policymaking. Recuperado de https://www.sustainabledevelopment.un.org/index.php?page=view \& type $=400 \& n r=2404$

Waters, C. N., Zalasiewicz, J., Summerhayes, C., Barnosky, A. D., Poirier, C., Gałuszka, A., ... Wolfe, A. P. (2016). The Anthropocene is functionally and stratigraphically distinct from the Holocene. Science, 351(6269). doi: $10.1126 /$ science.aad2622

Wilches-Chaux, G. (2008). Brújula, bastón y lámpara para trasegar los caminos de la educación ambiental. Ministerio de Ambiente, Vivienda y Desarrollo Territorial. Recuperado de https://www.comunidadandina.org/predecan/ doc/libros/brujula.pdf

World Wildlife Fund (WWF). (2018). Informe planeta vivo. 2018: apuntando más alto. Recuperado de https://www .awsassets.wwf.es/downloads/informe_planeta_vivo_2018.pdf

\section{Notas}

[1] Sustentable en el original.

[2] Sustentable en el original.

[3] Sustentabilidad en el original. 
[4] Sustentables en el original.

* Artículo de reflexión.

Este artículo fue elaborado durante el período 2017-2018, en la fase de formulación de la tesis para optar al título de doctora en Ciencias Naturales para el Desarrollo (Docinade), del Instituto Tecnológico de Costa Rica (Universidad Nacional de Costa Rica, Universidad Estatal a Distancia, Costa Rica), de la autora principal, titulada Plan de educación ambiental para la gestión integral de residuos sólidos del Sistema Universitario de Manizales, Colombia, financiada por la Universidad Católica de Manizales.

\section{Licencia Creative Commons CC BY 4.0}

Cómo citar este artículo: Calderón Cuartas, P. A., Osorio Viana, W., Naranjo Vasco, J. M., y Guzmán Hernández, T. de J. (2019). Formación de cultura ambiental desde el enfoque de ciclo de vida: una propuesta pedagógica para la sostenibilidad. Ambiente y Desarrollo, 23(44). https://doi.org/10.11144/Javeriana.ayd2 3-44.fcae 